\title{
Electric fields in crown ether complexation with neutral molecules
}

Mingwen Shi ${ }^{1}$, Sajesh Thomas ${ }^{1}$, Alison Edwards ${ }^{2}$, George Koutsantonis ${ }^{1}$, Bo Iversen ${ }^{3}$, Mark Spackman ${ }^{1}$ ${ }^{1}$ The University of Western Australia, Perth, Australia, ${ }^{2}$ Australian Nuclear Science and Technology Organisation, Sydney, Australia, ${ }^{3}$ Center for Materials Crystallgraphy, Aarhus University, Aarhus, Denmark E-mail: mingwen.shi@research.uwa.edu.au

18-crown-6 (18C6) and its derivatives are known for their broad binding affinities to a variety of cations and neutral molecules. Although crown ethers have been widely studied and used in cation transportation and separation, their complexation with neutral guest molecules is poorly studied in comparison. With the recent advances in supercomputing facilities, intense synchrotron radiation, spallation neutron source, novel detectors and cooling devices, simple but fundamental crown ether host-guest complexes have become amenable to the detailed theoretical and experimental investigations.

In recent years, we have focused extensively on studying the binding modes,[1] interaction energies,[2] and electrostatic properties of crown ether complexes with neutral guest molecules. Energy framework analysis revealed that the host-guest trimer or dimer is the building block in crystal formation.[1] The complexation free energies between $18 \mathrm{C} 6$ and small neutral molecules, calculated using G4/MP2 composite method, showed that the geometries of $18 \mathrm{C} 6$ host-guest complexes in the gas phase are remarkably similar to those in the solid state.[2] In our charge density study of the 18C6:(urea)5 complex, it was shown (in the attached Figure) that the magnitude of the urea dipole moment is dramatically enhanced, and its dipole direction is perpendicular to the local electrostatic potential contour across the molecule, and hence parallel to the electric field. What is more, the electric fields that urea molecules experience are much greater if they participate in 18C6:(guest)2 trimers. We have also studied asymmetric crown ether derivatives and systematically compared the electric fields that the guest molecule experiences in gas phase, solution state, pure crystalline state, $18 \mathrm{C} 6$ complex and dibenzo-18C6 complex. The results have shown the steady increase of the electric fields experienced by the neutral guest molecules.

This research is directly relevant to work by Boxer and colleagues[3] which measures the vibrational frequency change of characteristic functional groups $(-\mathrm{CN},-\mathrm{NO} 2,-\mathrm{CO})$ to derive the electric field in liquids and solids. This strategy is based on the vibrational Stark effect (VSE), but it has been overlooked that charge density analysis can derive electric fields in crystals in a more straightforward manner, and our results are directly comparable to those determined from VSE.

[1] Shi, M. W. et al. (2015). Cryst. Growth Des. 15, 5892-5900.

[2] Shi, M. W. et al. (2016). CrystEngComm. 18, 8653-8663.

[3] Suydam, I. T. et al. (2006), Science. 313, 200-204.

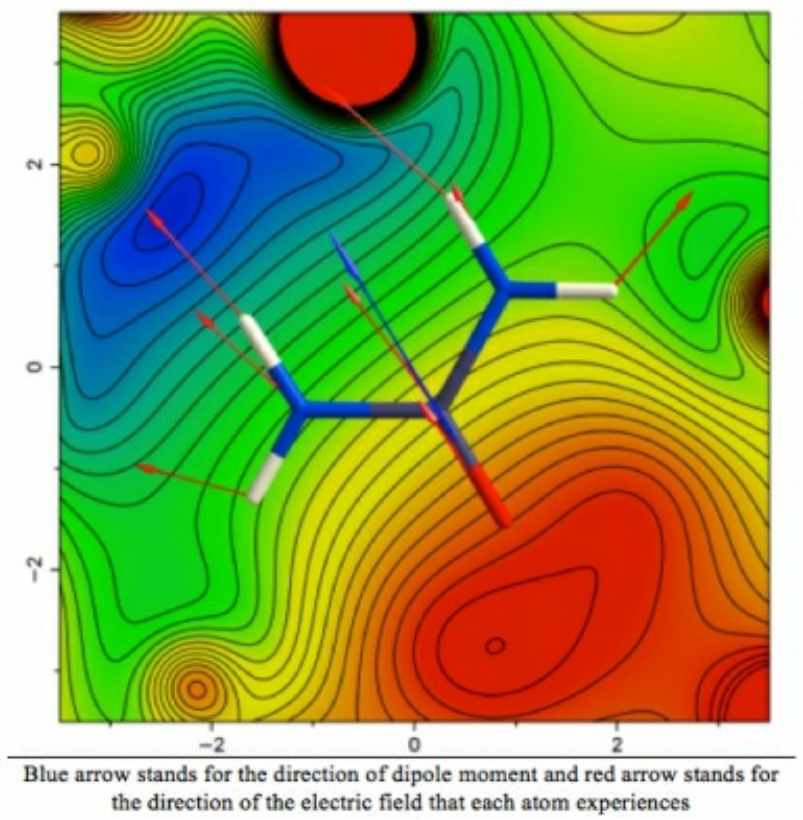

Keywords: dipole moment, electric field 